\title{
PAPR Reduction for 3FPP LTE OFDMA System
}

\author{
D.M. Motiur Rahaman \\ Rajshahi University of Engineering \& Technology, Bangladesh \\ *Corresponding Author: motiur_eee28@yahoo.com
}

Copyright (C 2013 Horizon Research Publishing All rights reserved.

\begin{abstract}
LTE (long term evolution) is come to meet our desired high speed data communication.LTE is the last step toward the 4th generation (4G) of radio technologies designed to increase the capacity and speed of mobile telephone networks. Where the current generation of mobile telecommunication networks are collectively known as $3 \mathrm{G}$ (for "third generation"), LTE is marketed as 4G. LTE uses Orthogonal Frequency Division Multiplexing (OFDM) for the downlink - that is, from the base station to the terminal. OFDMA meets the LTE requirement for spectrum flexibility and enables cost-efficient solutions for very wide carriers with high peak rates. However, one of the main drawbacks of the OFDMA modulation technique is the large peak-to-average power ratio (PAPR) of the transmitting signals. This high PAPR causes interference when the OFDM signals are passed through an amplifier which does not have enough linear range. Several PAPR reduction techniques such as magnitude clipping, block coding, and partial transmit sequence (PTS) and pulse shaping have been proposed to reduce the PAPR. In this paper, we evaluate a clipping based PAPR reduction technique. The complementary cumulative distribution function (CCDF) of the PAPR, which can be used to evaluate the PAPR reduction performance, is derived for OFDMA signals.
\end{abstract}

\section{Keywords 3GPP, LTE, OFDMA, CCDF, PAPR}

\section{Introduction}

At present time the demand on high data rate is increasing in order to support broadband services. Long term evolution (LTE) is a project of third generation partnership project (3GPP) and is an evolution to existing $3 \mathrm{G}$ technologies in order to increase the capacity and speed of mobile telephone networks. Current working assumptions in 3GPP LTE are to use single carrier frequency division multiple access (SCFDMA) for uplink and orthogonal frequency division multiplexing access (OFDMA) for downlink. SCFDMA is a promising technique for high data rate transmission that utilizes single carrier modulation and frequency domain equalization. Single carrier transmitter structure leads to keep the peak-to average power ratio (PAPR) as low as possible that will reduced the energy consumption as well as increase battery life time of the mobile to transmit signal from mobile terminal to the base station. SCFDMA has similar throughput performance and essentially the same overall complexity as OFDMA [2], [7], [8]. Orthogonal Frequency-Division Multiple Access (OFDMA) is a multi-user version of the popular Orthogonal Frequency Division Multiplexing (OFDM) digital modulation scheme. Multiple access is achieved in OFDMA by assigning subsets of subcarriers to individual users i.e. the enter channel is divided into many narrow sub-channel, which are transmitted in a parallel, thereby increasing the symbol duration and reducing the inter symbol-interference (ISI). Through OFDMA comprises many benefits (for high data rate) they also suffer many problems higher sensitivity to frequency offsets and phase noise high, envelop fluctuation in the time domain, leading to large PAPR. Therefore SCFDMA is used instead of OFDMA for uplink data transmission to reduce PAPR as well as increasing battery life time as well as reducing power consumption trends [14-16].

Recently, OFDMA has received much attention due to its applicability to high speed wireless multiple access communication systems. The problem associated with OFDM also suffers OFDMA inherently such as high PAPR. Some existing PAPR reduction techniques, which were originally designed for OFDM, process he whole data block as one unit, thus making downlink demodulation OFDMA systems more difficult since only part of the subcarriers in one OFDMA data block are of demodulated by each user's receiver. If downlink PAPR reduction is achieved by schemes designed for OFDM, each user has to process the whole data block and then demodulate the assigned subcarriers extract their own information. This introduces additional processing for each user's receiver. In the following we describe some modifications of PAPR reduction techniques for an OFDMA downlink. The PAPR problem for an OFDMA uplink is not as serious as that for downlink transmission since each user's transmitter modulates its data to only some of the subcarriers in each data block[11], [13].

Before OFDMA modulation the time domain signal is converted into the frequency domain by discrete Fourier transform (DFT) method therefore this is called DFT-spread 
OFDMA (DFTS-OFDM).Therefore the main benefit of DFTS-OFDM compare to scheme is reduced variations in the instantaneous transmit power, implying the possibility for increased power -amplifier efficiency, low-complexity high quality equalization in the frequency domain and flexible bandwidth assignment [12].

Rest of the paper is organized as follows: section 2.discussion of LTE system model, section 3. PAPR reduction techniques, section 4.simulation result, section 5.conclusion.

\section{LTE OFDMA System Model}

A simplified block diagram of the 3GPP LTE OFDMA transceiver is shown in figure 1

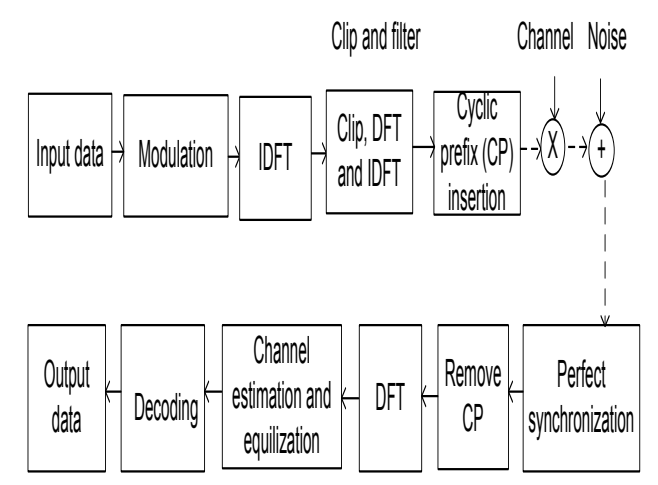

Figure 1. 3GPP LTE OFDMA system

A baseband modulator transmits the binary input to a multilevel sequences of complex number $x(k)$ in one of several possible modulation formats including, Binary phase shift keying (QPSK), and 16 level-QAM at the transmitter side. BPSK is the simplest form of phase shift keying (PSK. It uses two phases which are separated by $180^{\circ}$ and so can also be termed 2-PSK. It does not particularly matter exactly where the constellation points are positioned, and in this figure they are shown on the real axis, at $0^{\circ}$ and $180^{\circ}$. This modulation is the most robust of all the PSKs since it takes the highest level of noise or distortion to make the demodulator reach an incorrect decision. It is, however, only able to modulate at $1 \mathrm{bit} / \mathrm{symbol}$ and so is unsuitable for high data-rate applications when bandwidth is limited. These modulated symbols, are perform an N-point discrete Fourier transform (DFT) to produce a frequency domain representation [2]:

$$
\mathrm{S}_{1}(\mathrm{n})=\frac{1}{\sqrt{\mathrm{N}}} \sum_{\mathrm{k}=1}^{\mathrm{N}-1} \mathrm{x}(\mathrm{k}) \mathrm{e}^{\frac{(-\mathrm{j} 2 \pi \mathrm{kn})}{\mathrm{N}}}
$$

And inverse discrete Fourier transform for time domain expression:

$$
\mathrm{x}(\mathrm{k})=\frac{1}{\sqrt{\mathrm{N}}} \sum_{\mathrm{k}=0}^{\mathrm{N}-1} \mathrm{~S} 1(\mathrm{n}) \mathrm{e}^{\frac{(\mathrm{j} 2 \pi \mathrm{kn})}{\mathrm{N}}}
$$

where $\mathrm{k}$ is the sample index, $\mathrm{j}$ is the imaginary unit, $\mathrm{x}$ is the discrete symbol and $x(k)$ is the data symbol From IDFT time domain signal is passes for clipping and filtering. The clipping is followed by filtering to reduce out of band power. The DFT transform the clipped signal into frequency domain signal. The in-band frequency domain signals are passed to the second IDFT while out-of band signal components are null. Therefore it doesn't causes interference to the in-band OFDMA signal [1].Then cyclic prefix inserted and transmitted through the channel. In the same way at the receiver side the signal is receive and the cyclic pre fix is removed then this incoming signal is DFT and we get the base band signal after decoding.

Peak to average power ratio (PAPR) is a comparison of the peak power detected over a period of sample occurs over the same time period and defined as [8-10]:

$$
\text { PAPR }=\frac{\max _{0<\mathrm{m}<\mathrm{T}}|\mathrm{s}(\mathrm{m})|^{2}}{\frac{1}{\mathrm{TN}} \int_{0}^{\mathrm{TN}}|\mathrm{s}(\mathrm{m})|^{2} \mathrm{dm}}, \ldots . .
$$

Expression in decibels:

$$
\mathrm{PAPR}_{\mathrm{dB}}=10 \log _{10} \text { (PAPR) }
$$

Where $\mathrm{s}(\mathrm{m})$ is the transmitted signal, $\mathrm{T}$ is the symbol period of the transmitted signal One of the most frequently used performance measures for PAPR reduction techniques is cumulative distribution function (CDF). But in this paper we use, the complementary CDF (CCDF) is commonly used instead of the CDF. The CCDF of the PAPR denotes the probability that the PAPR of a data block exceeds a given threshold. There is another performance parameter bit error rate analysis. Although QPSK can be viewed as a quaternary modulation, it is easier to see it as two independent modulated quadrature carriers. With this interpretation, the even bits are used to modulate the in-phase component of the carrier, while the odd (or even) bits are used to modulate the quadrature-phase component of the carrier. BPSK is used on both carriers and they can be independently demodulated. Generally bit error rate of BPSK is lower than the QPSK.

\section{PAPR Reduction Technique}

Amplitude clipping is commonly used the Simplest technique for PAPR reduction. In a amplitude clipping the peak envelope of the input signal limits to a predetermined level or otherwise passes the input signal through without change that is:

$$
\mathrm{X}(\mathrm{y})=\mathrm{x}(\mathrm{y}), \quad \text { if }|x(y)| \leq W
$$

and

$$
\mathrm{W} \mathrm{e}^{\mathrm{i} \mathrm{q}(\mathrm{y})}, \text { if }|x(y)|>W
$$

where $\mathrm{q}(\mathrm{y})$ is the phase of transmitted signal $\mathrm{x}$. Without filtering noise produced due to amplitude clipping falls both in-band and out of band. Out-of-band radiation reduces spectral efficiency and in-band distortion cannot be reduced by filtering and results in error performance degradation. 
Though at first clipping and then filtering can reduce out of band radiation which may also cause some peak re-growth. As a result the signal after clipping and filtering will exceed the clipping level at some points. We can use iterative clipping-and-filtering operation to reduce overall peak re-growth in our desired level. When the signal converted into the time domain again, the signal is recovered with some error, though it is close to the original signal. An improvement can be made by repeating the above procedures.

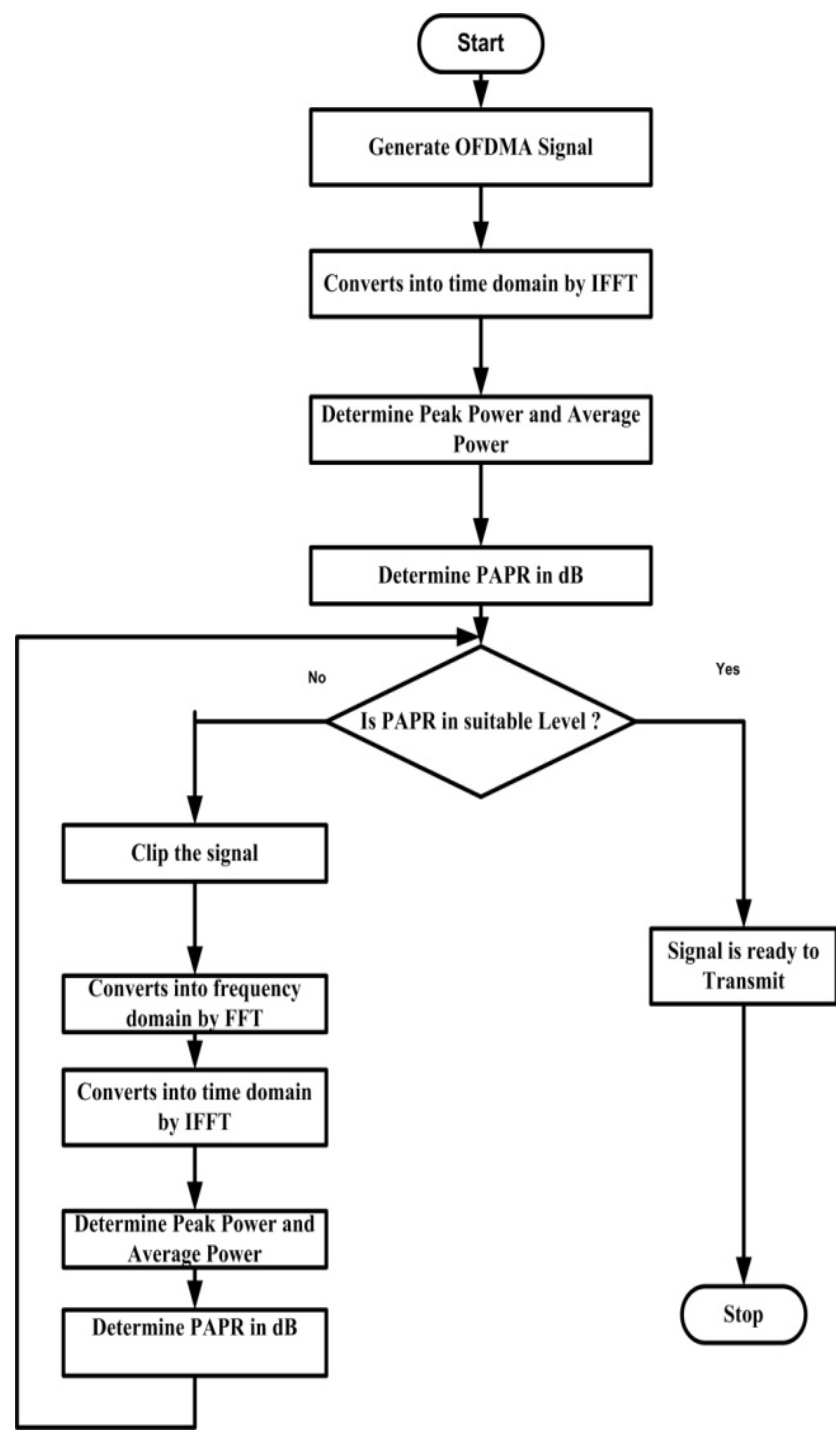

Figure 2. Flow chart for proposed scheme

In this section, we describe clipping technique. These techniques help fast PAPR reduction with lower no. of iterations. Each iteration steps shown is in fig. 2 (flow chart).

\section{Simulation Result}

Complementary cumulative distribution function (CCDF) of PAPR, which is the probability that PAPR is higher than a certain PAPR value PAPR0, is calculated by Monte Carlo simulation. The parameters used for the calculation of PAPR are illustrated in Table I

Table 1. The system parameters used for simulations

\begin{tabular}{|c|c|}
\hline SIZE OF OFDM Symbol & 256 \\
\hline $\begin{array}{c}\text { Interpolation factor (Oversampling } \\
\text { factor) }\end{array}$ & 2 \\
\hline SIZE OF FFT & 512 \\
\hline modulation & BPSK \\
\hline iteration & 8 \\
\hline Maximum symbol & $1 \mathrm{e}^{\wedge} 4$ \\
\hline
\end{tabular}

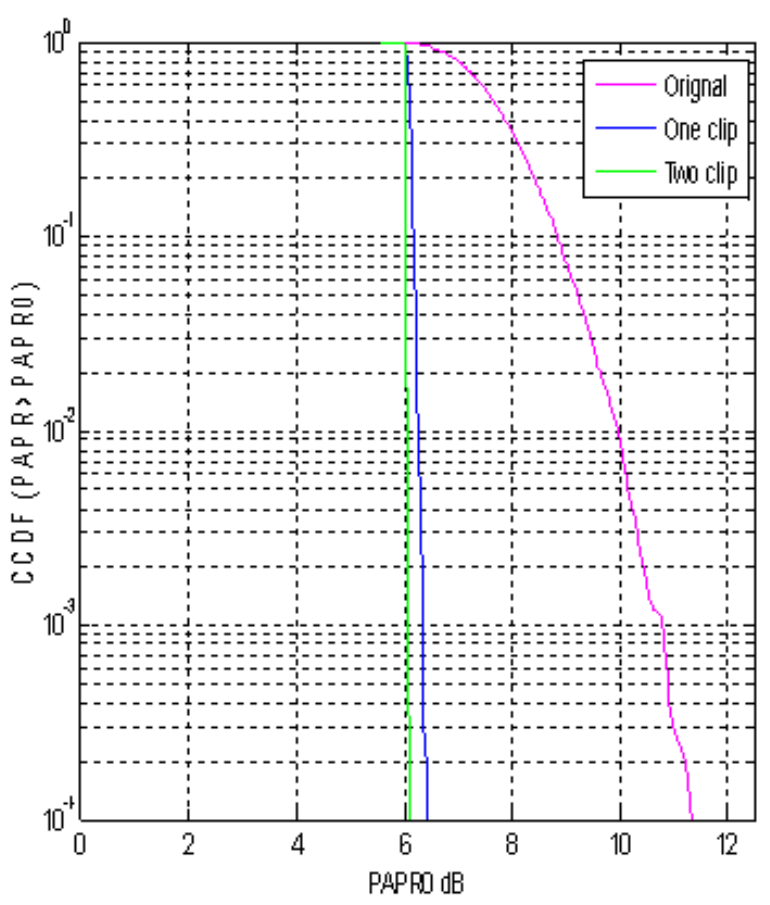

Figure 3. Comparison of CCDF of PAPR for LTE OFDMA using clipping method only

Now our interest to find the comparison of PAPR of 3GPP LTE OFDMA system between original OFDMA signal PAPR, PAPR after one clip of the OFDMA signal, PAPR after two clip of the OFDMA signal . From the fig. 3 it is clear that original OFDMA signal PAPR is greater than other . Further PAPR after one clip is greater than the two clip of the OFDMA signal. As the no number of clip is increasing PAPR of the OFDMA signal is is reduce but there is the main problem of higher order clipping some signal distortion caused in-band and out of band. To reduce signal distortion after clipping filtering is necessary.

Now our interest to find the comparison of PAPR of $3 \mathrm{GPP}$ LTE OFDMA system between original OFDMA signal PAPR, PAPR after one clip and filtering of the OFDMA signal, PAPR after two clip and filtering of the OFDMA signal . From the fig. 4 it is clear that original OFDMA signal PAPR is greater than other. Further PAPR after one clip and 
filtering is greater than the two clip and filtering of the OFDMA signal. As the no number of clip and filtering is increasing PAPR of the OFDMA signal is is reduce. Now we compare PAPR of OFDMA signal with clipping and filtering and without filtering.

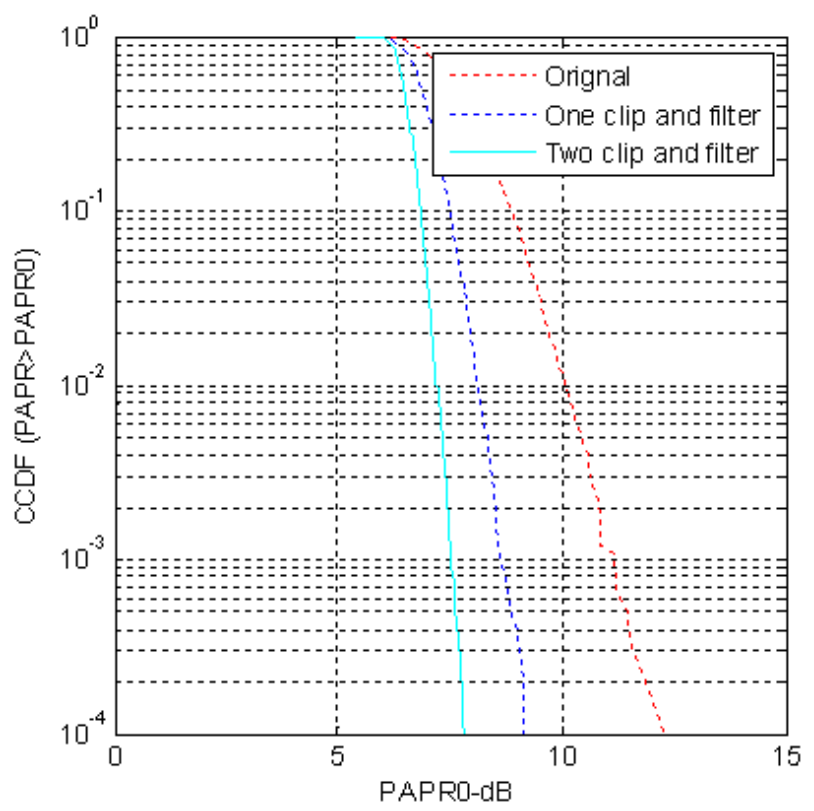

Figure 4. Comparison of CCDF of PAPR for LTE OFDMA using clipping and filtering method

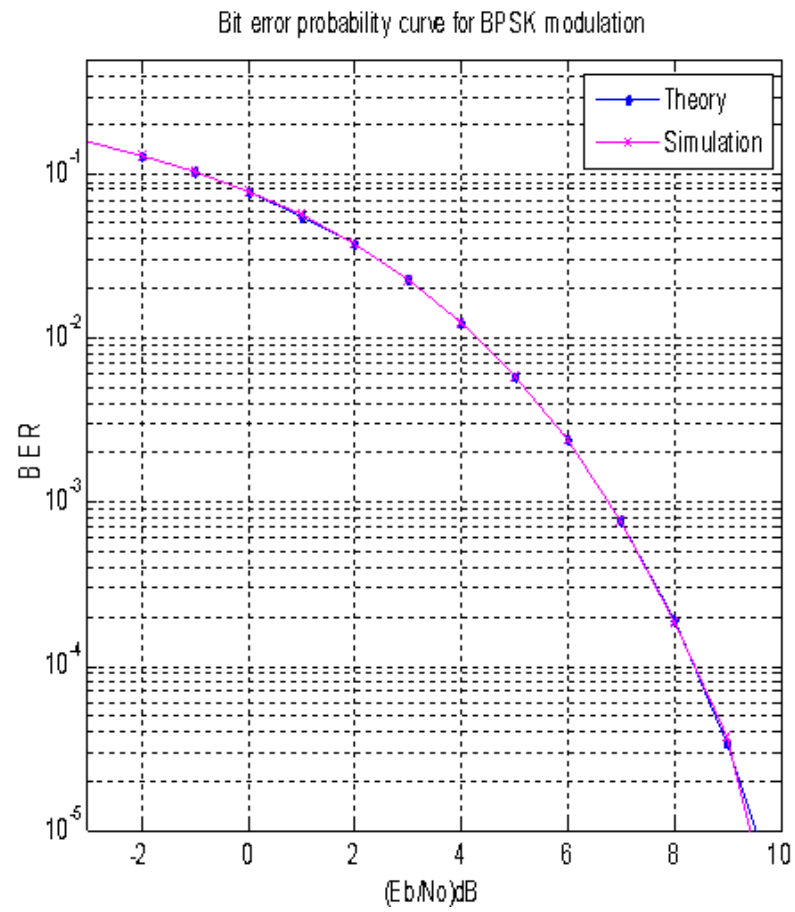

Figure 5. BER vs. SNR using ideal channel

The simulation result of this method is shown in figure 2 and 3. It can be observe that OFDM signal is has higher PAPR and after applying this method PAPR is reduced significantly. This PAPR is decreases as the number of clip and filtering is increased. Because the clipping is followed by filtering to reduce out of band power. The bit error rate (BER) is another important performance parameter in wireless communication system for quality measurement of recovered data. Next, we evaluate the BER performance for BPSK or QPSK modulation. Theoretical equation for bit error rate (BER) with Binary Phase Shift Keying (BPSK) modulation scheme in Additive White Gaussian Noise (AWGN) channel the BER results obtained using Mat lab simulation scripts show good agreement is shown in figure4(for ideal channel). Noise produced due to Amplitude clipping falls both in-band out of band. Out-of-band radiation reduces spectral efficiency and in-band distortion cannot be reduced by filtering and results in an error performance degradation. Though at first clipping and then filtering can reduce out-of band radiation which may also cause some peak re-growth. As a result the signal after clipping and filtering will exceed the clipping level at some points. We use iterative clipping-and-filtering operation to reduce overall peak re-growth in our desired level.

\section{Conclusion}

Multicarrier transmission is a very attractive technique for high-speed transmission over a dispersive communication channel. The PAPR problem is one of the important issues to be addressed in developing multicarrier transmission systems in LTE. In this article we describe PAPR reduction technique for multicarrier transmission. From the computer simulations we can come to the conclusion that this clipping method reduced the PAPR significantly as well as increase average transmitted power improve amplifier efficiency and reduced cost.

\section{REFERENCES}

[1] S. H. Han, J. H. Lee, "An overview of peak-to-average power ratio reduction techniques for multicarrier transmission," IEEE Personal Communications, vol. 12, pp.56 - 65. iss.2, April 2005.

[2] B. Karakaya, H.Arslan, and H. A. Cirpan, "Channel estimation for LTE uplink in high Doppler spread," Proc. WCNC, pp. 1126-1130, April 2008.

[3] T. S. Rappaport, Wireless communications principles and practice, Prentice Hall, 2nd Edition, 1998.

[4] S. K. Deng, M. Chao 'OFDM PAPR Reduction Using Clipping with Distortion Control" IEEE TRANSACTIONS ON COMMUNICATIONS, VOL. 55, NO. 1, JANUARY 2007.

[5] A. Venkataraman, H. Reddy, and Tolga M. Duman 'Space-Time Coded OFDM with Low PAPR', EURASIP Journal on Advances in Signal Processing. 23 February 2006.

[6] H. G. Myung, J. Lim, and D. J. Goodman, "Single carrier FDMA for uplink wireless transmission," IEEE Vehicular 
Technology Magazine, vol. 1, no. 3, pp. 30-38, September 2006.

[7] H.G. Myung, J .Lim, and D. J. Goodman,'Peak to average power ratio for single carrier FDMA signals," Proc. PIMRC, 2006.

[8] M. Salah, G. Abdel-Fadeel, and Zaki B. Nossair, Peak to Average Power Ratio Reduction in Single Carrier OFDMA Systems

[9] R. Alex. Panicker and Joseph M. Kahn, Fellow, IEEE, Algorithms for Compensation of Multimode FiberDispersion Using Adaptive Optics

[10] H. Sari,G. Karam and Jeanclaude, “An analysis of Orthogonal Frequency -Division Multiplexing for Mobile Radio Applications," Proc.IEEE VTC, pp. 1635-1639,June 1994

[11] J. Amastrong, "Peak to average power reduction for OFDM by repeated clipping and frequency domain filtering," Electronics letter, iss. 5, 2002.

[12] A. Goldsmith, "Wireless communications," Cambridge University Press, 2005.

[13] G.Yue and X. Wang, "A hybrid PAPR reduction scheme for coded OFDM," IEEE Wireless Commun., vol. 5, pp. 2712-2722, Oct. 2006

[14] H. Jafarkhani, "A quasi-orthogonal space-time code," IEEE Trans. Commun, vol. 49, pp. 1-4, Jan. 2001.

[15] J. Siew, R. J. Piechocki, A. Nix, and S. Armour, "A channel estimation method for MIMO-OFDM systems," in Proc. London Communications Symp. 2002-UCL, pp. 101-104, Sept. 2002.

[16] U. K. Kwon, G. H. Im, and E. S. Kim, "An iteration technique for recovering insufficient CP and clipped OFDM signals," IEEE Singal Processing Lett., vol. 14, pp. 317-320, May 2007. 